\title{
Gaussian pure states in quantum mechanics and the symplectic group
}

\author{
R. Simon* \\ Centrefor Theoretical Studies, Indian Institute of Science, Bangalore 560012 , India \\ E. C. G. Sudarshan ${ }^{\dagger}$ \\ Centerfor Particle Theory, The University of Texas, Austin, Texas 78712 \\ N. Mukunda \\ Centrefor Theoretical Studies and Department of Physics, Indian Institute of Science, Bangalore 560012, India
}

\begin{abstract}
Gaussian pure states of systems with $n$ degrees of freedom and their evolution under quadratic Hamiltonians are studied. The Wigner-Moyal technique together with the symplectic group $\mathrm{Sp}(2 n, \mathbb{R})$ is shown to give a convenient framework for handling these problems. By mapping these states to the set of $n \times n$ complex symmetric matrices with a positive-definite real part, it is shown that their evolution under quadratic Hamiltonians is compactly described by a matrix generalization of the Mobius transformation; the connection between this result and the "abcd law" of Kogel. nik in the context of laser beams is brought out. An equivalent Poisson-bracket description over a special orbit in the Lie algebra of $\mathbf{S p}(2 n, \mathbb{R})$ is derived. Transformation properties of a special class of partially coherent anisotropic Gaussian Schell-model optical fields under the action of $\operatorname{Sp}(4, \mathbb{R})$ first-order systems are worked out as an example, and a generalization of the "abcd law" to the partially coherent case is derived. The relevance of these results to the problem of squeezing in multimode systems is noted.
\end{abstract}

\section{INTRODUCTION}

It is a well-known fact in both classical and quantum mechanics that the dynamics of a system with a Hamiltonian quadratic in coordinates and momenta is closely related to a real symplectic group. Thus for such a system with $2 n$ phase-space variables, both the numerical Hamilton and the operator Heisenberg equations of motion are linear ordinary differential equations, whose solution involves a one-parameter group of matrices belonging to the defining representation of the group $\operatorname{Sp}(2 n, \mathbb{R})$. This solution represents a canonical transformation in the classical case, and a unitary transformation in quantum mechanics.

The simple dynamics of such systems leads, in quantum mechanics, to the study of a special class of wave functions which maintain their general form as they evolve in time in accordance with the Schrodinger equation. These wave functions are normalizable complex Gaussians, which are therefore completely determined by the means and variances of the coordinate and momentum operators. If one regards these means and variances as time-dependent parameters characterizing the wave function, then the Schrodinger equation for the latter leads in a direct way to an in general nonlinear system of evolution equations for the former. Such quantummechanical state functions have recently been given the distinctive name "Gaussons;" 1 and for a particular choice of quadratic Hamiltonian their evolution in time has been studied in detail. ${ }^{2}$ Both ordinary and squeezed ${ }^{3}$ coherent states are examples of "Gaussons."

It is clear that a corresponding treatment of classical systems of this type can be given at the level of the Hamilton-Jacobi equation.

We are interested in this paper in quantum-mechanical pure states with Gaussian wave functions [see Eq. (2.20) below for a precise definition], and the evolution of these states under the action of Hamiltonians quadratic in the canonical variables. Such states have been called, in a descriptive manner, "Gaussian pure states (GPS)" (or "Gaussons", ).

The fundamental significance of the real symplectic group $\operatorname{Sp}(2 n, \mathbb{R})$ in this context arises from the fact that it is the group of linear automorphisms of the canonical commutation relations among $n$ pairs of coordinatemomentum operators. It follows that the Hilbert space on which these operators act irreducibly carries a unitary representation of $\operatorname{Sp}(2 n, \mathbb{R})$. The infinitesimal generators for this representation are quadratic Hermitian expressions in the canonical variables, and these are possible Hamiltonians for the class of systems under consideration.

While elements of $\operatorname{Sp}(2 n, \mathbb{R})$ act on vectors by their representative unitary transformations, the action on operators is by conjugation. It has been shown elsewhere' that this action finds its most natural and direct expression when operators are described by their Wigner-Moyal representatives. It follows that this description is suited also for a discussion of the behavior of GPS's under $S p(2 n, \mathbb{R})$, since one can set up the pure state density operator for such a wave function and then pass to its Wigner-Moyal representative. ${ }^{6}$

The purpose of this paper is to provide a complete analysis of GPS's along these lines.' In particular the un- 
itary action of the most general symplectic transformation on a GPS density matrix can be displayed explicitly. We will see that Gausson states correspond one to one to points on a special orbit in the Lie algebra $\operatorname{sp}(2 n, \mathbb{R})$ of $\mathrm{Sp}(2 n, \mathbb{R})$ under the adjoint action. And the effect of an element of $\mathbf{S p}(2 n, \mathbb{R})$ on a GPS is exactly representable as a motion on this orbit. This motion in turn can be interpreted as a canonical transformation with respect to a classical phase-space structure that intrinsically belongs to the orbit. Thus the quantum-mechanical evolution of GPS's according to the Schrodinger equation is exactly equivalent to a classical canonical evolution of the parameters along this special orbit in $\operatorname{sp}(2 n, \mathbb{R})$. This statement is true for any choice of Hamiltonian provided only that it is quadratic.

The material of this paper is organized as follows. In Sec. II we introduce the symplectic group $\operatorname{Sp}(2 n, \mathbb{R})$ and its action on canonical coordinate and momentum operators, and its associated unitary representation. The Wigner-Moyal description of operators is then recalled, and its special features with respect to $\operatorname{Sp}(2 n, \mathbb{R})$ displayed. With this preparation, the family of GPS's is defined as a special subset of a more general family of operators with Gaussian configuration-space kernels. The problem of the unitary action of $\operatorname{Sp}(2 n, \mathbb{R})$ transformations on GPS's is posed. In Sec. III this problem is converted, with the use of Wigner-Moyal representatives, into a matrix-theoretical problem. It is shown that the problem can be solved by exploiting the properties of the very special orbit in the Lie algebra $\operatorname{sp}(2 n, \mathbb{R})$ of $\operatorname{Sp}(2 n, \mathbb{R})$. The result is that any element of $\operatorname{Sp}(2 n, \mathbb{R})$, via its associated unitary transformation, maps each GPS into another such state. The changes produced in the parameters of the state can be displayed in a rather elegant form. Section IV sets up the classical phase-space structure on the Lie algebra $\mathbf{s p}(2 n, \mathbb{R})$ orbit mentioned above, and shows that quantum-mechanical evolution of a GPS with a quadratic Hamiltonian operator is mirrored in a classical canonical evolution of the parameters along this orbit. A coordinate system for this orbit, which is both global and canonical, is displayed. Section V describes an application of the results of Secs. III and IV to a special class of partially coherent optical fields, and Sec. VI contains some concluding remarks.

\section{SYMPLECTICGROUP, WIGNER-MOYAL REPRESENTATIVES, AND GPS's}

We consider a quantum-mechanical system based on 2n Hermitian coordinate and momentum operators $\hat{q}_{r}, \hat{p}_{r}, r=1,2, \ldots, n$. It is convenient to set up a column vector $\hat{Q}$ with 2 n Hermitian operator entries as

$$
\hat{Q}=\left(\hat{q}_{1}, \ldots, \hat{q}_{n}, \hat{p}_{1}, \ldots, \hat{p}_{n}\right)^{T} .
$$

Then the canonical commutation relations can be compactly written as

$$
\begin{aligned}
& {\left[\hat{Q}_{a}, \hat{Q}_{b}\right]=i \beta_{a b}, \quad a, b=1,2, \ldots, 2 n} \\
& \underline{\beta}=\left[\begin{array}{cc}
\underline{Q}_{n \times n} & \underline{\mathbb{1}}_{n \times n} \\
-\underline{\mathbb{1}}_{n \times n} & \underline{\underline{Q}}_{n \times n}
\end{array}\right] .
\end{aligned}
$$

Eigenvalues of the $\hat{q}^{\prime}$ 's and $\hat{p}$ 's will be denoted by $q$ 's and $p$ 's, respectively. A real linear transformation taking the $\hat{Q}_{a}$ into Hermitian $\hat{Q}_{a}^{\prime}$ according to

$$
\hat{Q}_{a}^{\prime}=S_{a b} \hat{Q}_{b}
$$

will preserve the commutation relations (2.2) if and only if the real 2 n-dimensional matrix $\underline{S}=\left(S_{a b}\right)$ obeys

$$
\underline{S} \underline{\beta} \underline{S}^{T}=\underline{\beta} \text {. }
$$

This is the defining representation of the group $S p(2 n, \mathbb{R})$. We note that along with $S$, both $S^{-1}$ and $S^{T}$ belong to $\operatorname{Sp}(2 n, \mathbb{R})$. We shall be dealing with an irreducible representation of the $\hat{Q}$, which, for instance, in the Schrodinger representation using eigenvectors of the $\hat{q}$ appears as

$$
\begin{aligned}
& \left\langle q\left|\hat{q}_{r}\right| \psi\right\rangle=q_{r}\langle q \mid \psi\rangle=q_{r} \psi(q), \\
& \left\langle q\left|\hat{p}_{r}\right| \psi\right\rangle=-i \frac{\partial}{\partial q_{r}} \psi(q), \\
& \langle\psi \mid \psi\rangle=\int d^{n} q|\psi(q)|^{2}, \\
& \left\langle q \mid q^{\prime}\right\rangle=\delta^{(n)}\left(q-q^{\prime}\right) .
\end{aligned}
$$

The linear transformation (2.3) is then unitarily implementable, that is, there is a unitary operator $\mathcal{U}(S)$ for each $S \in \operatorname{Sp}(2 n, \mathbb{R})$ such that

$$
\begin{aligned}
& \mathcal{U}^{-1}(S) \hat{Q} \mathcal{U}(S)=S \hat{Q}, \\
& u\left(S_{1}\right) \mathcal{u}\left(S_{2}\right)=\mathcal{U}\left(S_{1} S_{2}\right)
\end{aligned}
$$

An element of $\operatorname{Sp}(2 n, \mathbb{R})$ close to the identity has the form

$$
S \simeq \mathbb{1}+\epsilon J, \quad|\epsilon|<1 .
$$

The symplectic condition (2.4) then leads to

$$
(\beta J)^{T}=\beta J \text {. }
$$

Thus the infinitesimal generators of the defining representation of $\operatorname{Sp}(2 n, \mathbb{R})$ are in one-to-one correspondence with real symmetric $2 n \times 2 n$ matrices: One passes from the latter to the former by left multiplication by $\beta$. For an $S$ of the form $(2.71, \mathcal{U}(S)$ has the expression

$$
\begin{aligned}
& u(S) \simeq 1-i \epsilon X(J), \\
& X(J)=-\frac{1}{2} \hat{Q}^{T} \beta J \hat{Q}
\end{aligned}
$$

The basic properties of the Hermitian generators $X(J)$ of the unitary representation $\mathcal{U}(S)$ of $\mathrm{Sp}(2 n, \mathbb{R})$ are

$$
\begin{aligned}
& -i[\hat{Q}, X(J)]=J \hat{Q}, \\
& -i\left[X(J), X\left(J^{\prime}\right)\right]=X\left(\left[J, J^{\prime}\right]\right)
\end{aligned}
$$

As $J$ goes over the Lie algebra $\operatorname{sp}(2 n, \mathbb{R})$ of $\operatorname{Sp}(2 n, \mathbb{R})$, $X(J)$ goes over all Hermitian quadratic expressions in $\hat{q}_{r}$ and $\hat{p}_{r}$.

We see from Eq. (2.6) that the action of $\operatorname{Sp}(2 n, \mathbb{R})$ on the $\hat{q}$ and $\hat{p}$ is by conjugation. This equation is analogous to the solution of the Heisenberg equations of motion in quantum mechanics. In contrast, keeping in mind the Schrodinger picture evolution of a density operator, we 
define the action on such operators $\hat{\Gamma}$ by

$$
\hat{\Gamma}^{\prime}=\mathcal{U}(S) \hat{\Gamma} \mathcal{U}^{-1}(S) .
$$

It is natural to try to express this operator action in a classical numerical form. ${ }^{8}$ This is achieved by going to the Wigner-Moyal representative of $\hat{\Gamma}$, which is a function of $2 \mathrm{n}$ classical real variables $q_{r}, p_{r}, r=1,2, \ldots, n$. Similar to the definition of $\hat{Q}$ in Eq. (2.1), we define a numerical column vector $\theta$ as -

$$
Q=\left(q_{1}, \ldots, q_{n}, p_{1}, \ldots, p_{n}\right)^{T} .
$$

The Wigner-Moyal representative $W(Q)$ of the operator $\hat{\Gamma}$ is defined as the partial Fourier transform of the coordinate-space kernel $\Gamma\left(q ; q^{\prime}\right)$ of $\hat{\Gamma}:^{6}$

$$
W(Q)=(2 \pi)^{-n} \int d^{n} q^{\prime} \Gamma\left(q-\frac{1}{2} q^{\prime} ; q+\frac{1}{2} q^{\prime}\right) \exp \left(i p^{T} q^{\prime}\right),
$$

$\Gamma\left(q ; q^{\prime}\right)=\left\langle q|\hat{\Gamma}| q^{\prime}\right\rangle$.

Then we find ${ }^{5}$

$$
\hat{\Gamma}^{\prime}=\mathcal{U}(S) \hat{\Gamma} \mathcal{U}^{-1}(S)=W^{\prime}(Q)=W\left(S^{-1} Q\right) .
$$

In addition to the linear homogeneous transformations (2.3) of $\hat{Q}$, we also need to deal with translations in $\hat{Q}$ by c-numbers. These are generated by the displacement operators familiar from the theory of coherent states. ${ }^{3}$ For any numerical $\mathrm{Q}$, with entries $q_{0 r}, p_{0 r}$ we define the unitary displacement operator

$$
D(\mathrm{Q},)=\exp \left(i \hat{\boldsymbol{Q}}^{T} \beta Q_{0}\right)=\exp \left(i \underline{p}{ }_{0}^{T} \underline{\hat{q}}-i \underline{q}{ }_{0}^{T} \underline{\hat{p}}\right) .
$$

The properties with respect to $\hat{Q}$ and $\mathcal{U}(S)$ relevant to this discussion

$$
\begin{aligned}
& D^{-1}\left(Q_{0}\right) \hat{Q} D\left(Q_{0}\right)=\hat{Q}+Q_{0}, \\
& \mathcal{U}^{-1}(S) D\left(Q_{0}\right) \mathcal{U}(S)=D\left(S^{-1} Q_{0}\right) .
\end{aligned}
$$

These relations correspond to a unitary representation of the semidirect product $\mathrm{g}^{\mathrm{g}}$ of $\mathrm{Sp}(2 \mathrm{n}, \mathbb{R})$ with the group of translations $T_{2 n}$. Conjugation of a general $\hat{\Gamma}$ by $D\left(Q_{0}\right)$ alters $\Gamma\left(q ; q^{\prime}\right)$ and $W(Q)$ in the following ways:

$$
\begin{aligned}
\hat{\Gamma}^{\prime} & =D\left(Q_{0}\right) \hat{\Gamma} D^{-1}\left(Q_{0}\right)=\Gamma^{\prime}\left(q ; q^{\prime}\right) \\
& =\Gamma\left(q-q_{0} ; q^{\prime}-q_{0}\right) \exp \left[\underline{i p}{ }_{0}^{T}\left(q-\underline{q}^{\prime}\right)\right]=W^{\prime}(Q) \\
& =W\left(Q-Q_{0}\right) .
\end{aligned}
$$

We shall be interested later in certain Hermitian operators $\hat{\Gamma}$ which have unit trace and whose kernels $\Gamma\left(q ; q^{\prime}\right)$ are Gaussian. The most general $\hat{\Gamma}$ of this type can be parametrized by three real $\mathrm{n} \times \mathrm{n}$ matrices $\underline{\underline{L}}, \underline{M}$, and $\underline{K}$, of which the first two are symmetric

$$
\begin{aligned}
\left\langle q\left|\hat{\Gamma}_{L, M, K}\right| q^{\prime}\right\rangle & =\Gamma_{L, M, K}\left(q ; q^{\prime}\right) \\
& =(2 / \pi)^{n / 2}(\operatorname{det} \underline{L})^{1 / 2} \exp \left[-\underline{q}^{T} \underline{L} \underline{q}-\underline{q}^{\prime} \underline{L}^{\prime} \underline{q}^{\prime}-\frac{1}{2}\left(\underline{q}-\underline{q}^{\prime}\right)^{T} \underline{M}\left(\underline{q}-\underline{q}^{\prime}\right)+\frac{1}{2} i\left(\underline{q}-\underline{q}^{\prime}\right)^{T} \underline{K}\left(\underline{q}+\underline{q}^{\prime}\right)\right] .
\end{aligned}
$$

We have restricted the expression in the exponent to be homogeneous quadratic in $q$ and $q^{\prime}$. We further require both $\underline{L}$ and $\underline{L}+\underline{M}$ to be positive definite, ensuring, respectively, that the trace is finite and that the integral occurring in Eq. (2.13) can be carried out. The normalization constants in the definition (2.18) have in fact been chosen to ensure unit trace. The Wigner-Moyal representative of $\hat{\Gamma}_{L, M, K}$ is

$$
\begin{aligned}
& W_{L, M, K}(Q)=\pi^{-n}[\operatorname{det} \underline{L} / \operatorname{det}(\underline{L}+\underline{M})]^{1 / 2} \exp \left(-Q^{T} \underline{G} Q\right), \\
& \underline{G}=\left[\begin{array}{cc}
\underline{C} \\
\underline{B}
\end{array}\right], \\
& A=2 \underline{L}+\frac{1}{2} \underline{K}^{T}(\underline{L}+\underline{M})^{-1} \underline{K}, \\
& \underline{B}=\frac{1}{2}(\underline{L}+\underline{M})^{-1}, \\
& \underline{C}=-\frac{1}{2} \underline{K}^{T}(\underline{L}+\underline{M})^{-1} .
\end{aligned}
$$

We see that it is completely characterized by a real symmetric (positive-definite) $2 \mathrm{n} \times 2 \mathrm{n}$ matrix $\underline{G}$ formed out of $\underline{L}, \underline{M}$, and $\underline{K}$.

With these preliminaries and notations established, we can define a general GPS. It is parametrized by a column vector $Q_{0}$, and two real symmetric $\mathrm{n} \times n$ matrices $\underline{U}, \underline{V}$ of which the former is positive definite. We combine $U$ and $\boldsymbol{V}$ into a complex symmetric matrix $\underline{\boldsymbol{Z}}=\underline{\boldsymbol{V}}-\boldsymbol{i} \underline{\underline{U}}$, and denote the GPS by $\left|\psi_{\boldsymbol{Z}, \mathbf{Q}_{\mathbf{0}}}\right\rangle$. Its wave function is

$$
\begin{aligned}
\left\langle q \mid \psi_{Z, Q_{0}}\right\rangle \equiv & \psi_{Z, Q_{0}}(q) \\
= & \pi^{-n / 4}(\operatorname{det} \underline{U})^{1 / 4} \\
& \quad \times \exp \left[-\frac{1}{2}\left(\underline{q}-\underline{q}_{0}\right)^{T}(\underline{U}+i \underline{V})\left(\underline{q}-\underline{q}_{0}\right)\right. \\
& \left.\quad+i \underline{p} \underline{0}_{0}^{T} \underline{q}\right] .
\end{aligned}
$$

This wave function is normalized to unity, and what appears in the exponent is the most general at most quadratic complex expression in $q$. It is normalizability that requires that $\mathbf{g}$ be positive definite.

From the point of view of classical beam optics, it is useful to note that Eq. (2.20) is a multidimensional generalization of coherent Gaussian (laser) beams. We are speaking here of coherent beams as opposed to partially coherent beams in classical optics, and this should not be confused with the quantum-mechanical coherent states wherein $\underline{U}$ would be the identity matrix and $\underline{V}$ the null matrix. In the beam case with the beam assumed to be propagating in the $x_{3}$ direction, $q=\left(x_{1}, x_{2}\right)$ is a twodimensional vector in the transverse plane with $\psi(q)$ and $|\psi(q)|^{2}$ giving, respectively, the complex field amplitude distribution and the intensity distribution in that plane. If we consider Gaussian beams with general astigmatism, then $\underline{U}$ and $\underline{V}$ are real symmetrix $2 \times 2$ matrices wirh $\underline{U}$ positive definite to ensure finiteness of the integrated intensity (normalizability) over the transverse plane. In the isotropic or rotationally invariant case (absence of astig. 
matism) $U$ is any positive constant times the unit matrix and $\underline{V}$ an arbitrary real constant times the unit matrix. In either case the paraxial propagation equation of classical wave optics gives the evolution of the beam from one transverse plane to another transverse plane. It turns out that the Gaussian form of $\psi(q)$ is preserved under this paraxial propagation, and hence the effect of propagation about the $x_{3}$ axis is simply to make the $2 \times 2$ matrices $\underline{U}$ and $\underline{V}$ evolve as functions of $\boldsymbol{x}_{3} \cdot{ }^{10}$

Our aim now is to show that these states $(2.20)$ transform in a simple way amongst themselves under action by $\mathcal{U}(S)$ for any $S \in \operatorname{Sp}(2 n, \mathbb{R})$. To this end we first calculate the kernel of the pure-state density operator $\hat{\rho}_{\mathbf{Z}, \boldsymbol{Q}_{0}}$ determined by $\left|\psi_{\boldsymbol{Z}, Q_{0}}\right\rangle$. After some simple algebraic manipulations, it is shown that this kernel is expressible in terms of $\Gamma_{L, M, K}\left(q ; q^{\prime}\right)$ for special choices of parameters and arguments

$$
\begin{aligned}
\hat{\rho}_{Z, Q_{0}}=\left|\psi_{Z, Q_{0}}\right\rangle\left\langle\psi_{Z, Q_{0}}\right| & \\
\rho_{Z, Q_{0}}\left(q ; q^{\prime}\right)= & \Gamma_{U / 2,0,-V}\left(q-q_{0} ; q^{\prime}-q_{0}\right) \\
& \times \exp \left[\underline{i p}{ }_{0}^{T}\left(\underline{q}-\underline{q}^{\prime}\right)\right] .
\end{aligned}
$$

Comparing this with Eq. (2.17) it is clear that the $Q_{0}$ dependence separates completely from the $Z$ dependence

$$
\begin{aligned}
& \hat{\rho}_{Z, Q_{0}}=D\left(Q_{0}\right) \hat{\rho}_{Z} D^{-1}\left(Q_{0}\right), \\
& \hat{\rho}_{Z}=\hat{\Gamma}_{U / 2,0,-V} .
\end{aligned}
$$

At the state-vector level this separation brings in an extra phase factor

$$
\begin{aligned}
& \left|\psi_{Z, Q_{0}}\right\rangle=e^{(i / 2) \underline{\underline{p}}_{0}^{T} \underline{q}_{0}} D\left(Q_{0}\right)\left|\psi_{Z}\right\rangle, \\
& \psi_{Z}(q)=\pi^{-n / 4}(\operatorname{det} \underline{U})^{1 / 4} \exp \left[-\frac{1}{2} \underline{q}^{T}(\underline{U}+i \underline{\underline{V}}) q\right] .
\end{aligned}
$$

This separation of $\mathbf{Z}$ and $Q_{0}$ is maintained under action by $\mathcal{U}(S)$ for any $S \in \mathrm{Sp}(2 n, \mathbb{R})$. In fact, on account of Eq. (2.16)we can see quite generally that if two operators $\widehat{\Gamma}_{0}$ and $\hat{\Gamma}$ are related by a displacement operator $D\left(Q_{0}\right)$,

$$
\hat{\Gamma}=D\left(Q_{0}\right) \hat{\Gamma}_{0} D^{-1}\left(Q_{0}\right),
$$

then their transforms by $\mathcal{U}(S)$ are similarly related:

$$
\begin{aligned}
& \hat{\Gamma}_{0}^{\prime}=\mathcal{U}(S) \hat{\Gamma}_{0} \mathcal{U}^{-1}(S), \\
& \hat{\Gamma}^{\prime}=\mathcal{U}(S) \hat{\Gamma}^{-1}(S)=D\left(Q_{0}^{\prime}\right) \hat{\Gamma}_{0}^{\prime} D^{-1}\left(Q_{0}^{\prime}\right), \\
& Q_{0}^{\prime}=S Q_{0} .
\end{aligned}
$$

In terms of Wigner-Moyal representatives, this means that if $W_{0}$ and $W$, corresponding to $\hat{\Gamma}_{0}$ and $\hat{\Gamma}$, respectively, are related by an initial phase-space displacement $Q_{0}$, then $S \in \operatorname{Sp}(2 n, \mathbb{R})$ maps them into $W_{0}^{\prime}$ and $W^{\prime}$ which are related by a transformed displacement $Q_{0}^{\prime}$ :

$$
\begin{aligned}
& W(Q)=W_{0}\left(Q-Q_{0}\right), \\
& W_{0}^{\prime}(Q)=W_{0}\left(S^{-1} Q\right), \\
& W^{\prime}(Q)=W\left(S^{-1} Q\right)=W_{0}\left(S^{-1} Q-Q_{0}\right)=W_{0}^{\prime}\left(Q-Q_{0}^{\prime}\right)
\end{aligned}
$$

The change of $Q$, to $Q_{0}^{\prime}$ is completely independent of $W_{0}(Q)$ and its changes. In dealing with GPS's we can therefore restrict ourselves to $\left|\psi_{Z}\right\rangle$ and study their $\mathrm{Sp}(2 n, \mathbb{R})$ transformation properties.

The key point now is to see that for any $S \in \operatorname{Sp}(2 n, \mathbb{R})$, $\mathcal{U}(S)$ maps $\left|\psi_{Z}\right\rangle$ into $\left|\psi_{Z^{\prime}}\right\rangle$ for a suitable $Z^{\prime}$, and to display the transformation law taking $\boldsymbol{Z}$ to $\boldsymbol{Z}^{\prime}$. Of course, one must make sure that $Z^{\prime}$ is a member of the same family of matrices to which $\boldsymbol{Z}$ belongs. For these purposes it is most convenient to work with the WignerMoyal representative of $\hat{p}_{Z}$, which is, using Eqs. (2.19) and (2.22),

$$
\begin{aligned}
& W_{Z}(Q)=\pi^{-n} \exp \left[-\underline{Q}^{T} \underline{G}(\underline{U}, \underline{V}) \underline{Q}\right], \\
& \left.\underline{G}(\underline{U}, \underline{V})=\left(\begin{array}{cc}
\underline{\underline{1}}_{n \times n} & \underline{\underline{0}}_{n \times n} \\
\underline{V} & \underline{\mathbf{1}}_{n \times n}
\end{array}\right)^{T}\left|\begin{array}{cc}
\underline{U} & \underline{\underline{0}}_{n \times n} \\
\underline{\underline{0}}_{n \times n} & \underline{U}^{-1}
\end{array}\right| \begin{array}{cc}
\underline{\underline{I}}_{n \times n} & \underline{\underline{0}}_{n \times n} \\
\underline{\boldsymbol{V}} & \underline{\mathbb{1}}_{n \times n}
\end{array}\right] \\
& =\left(\begin{array}{cc}
\underline{U}+\underline{V U}^{-1} \underline{V} & \frac{V U}{U^{-1}} \\
\underline{U}^{-1} \underline{V} & \underline{U}^{-1}
\end{array}\right) .
\end{aligned}
$$

According to Eq. (2.14), if $\mathcal{U}(S)$ maps $\hat{\rho}_{Z}$ to $\hat{\rho}^{\prime}$, the Wigner-Moyal representative $W^{\prime}(Q)$ of $\hat{\rho}^{\prime}$ is obtained from $W_{Z}(Q)$ by a linear transformation on the arguments:

$$
\begin{aligned}
& \hat{\rho}^{\prime}=\mathscr{U}(S) \hat{\rho}_{Z} \mathcal{U}^{-1}(S): \\
& W^{\prime}(Q)=W_{Z}\left(S^{-1} Q\right)=\pi^{-n} \exp \left(-\underline{Q}^{T} \underline{G}^{\prime} \underline{Q}\right), \\
& \underline{G}^{\prime}=\left(\underline{S}^{-1}\right)^{T} \underline{G}(\underline{U}, \underline{V}) \underline{S}^{-1} .
\end{aligned}
$$

(One may be tempted ${ }^{5}$ to avoid the excessive appearance of $S^{-1}$ in the above expressions and hereafter, by altering the conventions so far adopted. However, we choose not to do so, in order to be consistent with established usage in optics especially in relation to the "abcd law" which we generalize in Sec. V.) Our task is to show that there are real symmetric $n \times n$ matrices $\underline{U}^{\prime}$ and $\underline{V}^{\prime}$, the former being positive definite, such that

$$
\underline{G}^{\prime}=\underline{G}\left(\underline{U}^{\prime}, \underline{V}^{\prime}\right),
$$

and to exhibit $\underline{U}^{\prime}$ and $\underline{V}^{\prime}$ in terms of $\underline{S}, \underline{U}$, and $\underline{V}$. We take up these questions in Sec. III.

To conclude this section we return briefly to the complete GPS $\left|\psi_{Z, Q_{0}}\right\rangle$. By Eqs. (2.17), (2.22), and (2.27), the Wigner-Moyal representative of the density operator $\hat{\rho}_{Z, Q_{0}}$ is

$$
\begin{aligned}
W_{Z, Q_{0}}(Q) & =W_{Z}\left(Q-Q_{0}\right) \\
& =\pi^{-n} \exp \left[-\left(\underline{Q}-\underline{Q}_{0}\right)^{T} \underline{G}(\underline{U}, \underline{V})\left(\underline{Q}-\underline{Q}_{0}\right)\right] .
\end{aligned}
$$

Therefore using the Weyl rule for associating a $c$-number function of $Q$ with each operator function of $\hat{Q}$, we get the following values for the means and variances of the $\widehat{q}$ 's and $\hat{p}$ 's in a general GPS: 


$$
\begin{aligned}
\left\langle\psi_{Z, Q_{0}}\left|\hat{Q}_{a}\right| \psi_{Z, Q_{0}}\right\rangle= & \int d^{2 n} \hat{Q} Q_{a} W_{Z, Q_{0}}(Q)=Q_{0 a} \\
\left\langle\psi_{Z, Q_{0}}\left|\hat{Q}_{a} \hat{Q}_{b}\right| \psi_{Z, Q_{0}}\right\rangle= & \int d^{2 n} Q\left(Q_{a} Q_{b}+\frac{1}{2} i \beta_{a b}\right) \\
& \times W_{Z, Q_{0}}(Q) \\
= & \frac{1}{2} i \beta_{a b}+Q_{0 a} Q_{0 b} \\
& +\frac{1}{2}\left[\underline{G}^{-1}(\underline{U}, \underline{V})\right]_{a b},
\end{aligned}
$$

that is,

$$
\begin{aligned}
& \left\langle\psi_{Z, Q_{0}}\left|\left(\underline{\hat{Q}}-\underline{Q}_{0}\right)_{a}\left(\underline{\hat{Q}}-\underline{Q}_{0}\right\rangle_{b}\right| \psi_{Z, Q_{0}}\right\rangle \\
& =\frac{1}{2} i \beta_{a b}+\frac{1}{2}\left[\underline{G}\left(\underline{U}^{-1},-\underline{V}\right)\right]_{a b} .
\end{aligned}
$$

Naturally, these means and variances determine $\hat{\rho}_{\boldsymbol{Z}, Q_{0}}$ completely.

We conclude this section with the following observations. GPS's in the context of systems with one and two degrees of freedom have been studied in great detail in Ref. 4. But our analysis in the present paper is for a system with an arbitrary number of degrees of freedom, $n$. Thus, our results in this preparatory section when specialized to $n=1,2$ should be expected to give results consistent with the results of Ref. 4. In particular, compare our Eq. (2.20) and Eq. (2.31), respectively, with Eq. (3.2.1) and Eq. (3.3.19b) of Ref. 4. It should be added, however, that the principal results of this paper are the matrix generalized "abcd law" [Eq. (3.27) below] and the quantum description through classical Hamiltonian dynamics on a special orbit in the Lie algebra $\operatorname{sp}(2 n, \mathbb{R})$ of $\operatorname{Sp}(2 n, \mathbb{R})$ presented in Sec. IV, both of which are new and go well beyond the results in Ref. 4 even for $\mathrm{n}=1,2$.

\section{ACTION OF SYMPLECTIC TRANSFORMATIONS ON GPS's}

Each GPS density operator (with $Q_{0}=0$ which is hereafter assumed) has been seen to be uniquely characterized by a real symmetric positive-definite $2 n \times 2 n$ matrix $\underline{G}(\underline{U}, \underline{V})$. We examine the properties of the family of matrices arising in this way. On account of the symmetry, Eq. (2.8) shows that each such matrix is uniquely associated with an $\operatorname{sp}(2 n, \mathbb{R})$ matrix $\underline{J}(\underline{U}, \underline{V})$ :

$$
\underline{J}(\underline{U}, \underline{V})=\underline{B} \underline{G}(\underline{U}, \underline{V}) \in \mathrm{sp}(2 n, \mathbb{R}) .
$$

Thus we have a property at the Lie-algebra level. What is perhaps surprising and somewhat unusual is that the matrices $\underline{G}(\underline{U}, \underline{V})$ are also elements of $\mathbf{S p}(2 n, \mathbb{R})$, i.e., they belong to the defining representation of this group. This can be seen directly by checking that, since $\underline{U}$ and $\underline{V}$ are symmetric, Eq. (2.4) is satisfied by $\underline{G}(\underline{U}, \underline{V})$. A more useful way of expressing this property is to write

$$
\begin{aligned}
& \underline{G}(\underline{U}, \underline{V})=\left[\underline{S}^{-1}(\underline{U}, \underline{V})\right]^{T} \underline{S}^{-1}(\underline{U}, \underline{V}) \in \operatorname{Sp}(2 n, \mathbb{R}), \\
& \underline{S}(\underline{U}, \underline{V})=\left(\begin{array}{cc}
\underline{\mathbb{1}}_{n \times n} & \underline{0}_{n \times n} \\
-\underline{V} & \underline{\mathbb{1}}_{n \times n}
\end{array}\right]\left(\begin{array}{cc}
\underline{U}^{-1 / 2} & \underline{0}_{n \times n} \\
\underline{0}_{n \times n} & \underline{U}^{1 / 2}
\end{array}\right) \in \operatorname{Sp}(2 n, \mathbb{R}) .
\end{aligned}
$$

[Here we recall that if $\underline{S}$ belongs to $\operatorname{Sp}(2 n, \mathbb{R})$, so does
$\underline{S}^{T}$.]

From the identification (3.1) in terms of Lie-algebra elements, we see that as $S$ ranges all over $\operatorname{Sp}(2 n, \mathbb{R})$ the passage from $\underline{G}(\underline{U}, \underline{V})$ to $\underline{G}^{\prime}$ given in Eq. (2.28) corresponds to conjugation of $\underline{\underline{J}}(\underline{\underline{U}}, \underline{\underline{V}})$ and so to passing from $\underline{J}(\underline{U}, \underline{V})$ to all other elements $\underline{\underline{J}}^{\prime}$ on the orbit of $\underline{\boldsymbol{J}}(\underline{U}, \underline{\boldsymbol{V}})$ defined by the adjoint action

$$
\begin{aligned}
& \underline{G}^{\prime}=\left(\underline{S}^{-1}\right)^{T} \underline{G}(\underline{U}, \underline{V}) \underline{S}^{-1}, \\
& \underline{J}^{\prime}=\underline{\beta}^{\prime}=\underline{J}^{\prime}=\underline{S} \underline{J}(\underline{U}, \underline{V}) \underline{S}{ }^{-1} .
\end{aligned}
$$

At the same time Eq. (3.2) shows that $\underline{G}(\underline{U}, \underline{V})$ is the symmetric transform (or better symplectic transform), via $\underline{S}(\underline{U}, \underline{V})$, of the unit matrix

$$
\begin{aligned}
& \underline{G}(\underline{U}, \underline{V})=\left[\underline{S}-{ }^{1}(\underline{U}, \underline{V})\right]^{T} \underline{G}{ }^{(0)} \underline{S}-{ }^{1}(\underline{U}, \underline{V}), \\
& \underline{G}^{(0)}=\underline{G}\left(\underline{\mathbb{I}}_{n \times n}, \underline{\underline{O}}_{n \times n}\right)=\underline{\mathbb{I}}_{2 n \times 2 n} .
\end{aligned}
$$

Equivalently, $\underline{J}(\underline{U}, \underline{V})$ arises from $\underline{J}^{(0)}=\underline{J}\left(\underline{\underline{1}}_{n \times n}, \underline{0}_{n \times n}\right)$ $=\underline{\beta}$ by adjoint action with $\underline{S}(\underline{U}, \underline{V})$. Let $\mathcal{O}$ be the orbit of $\beta$ in $\operatorname{sp}(2 n, \mathbb{R})$,

$$
\mathcal{O}=\left\{\underline{S}_{\underline{S}} \underline{S}^{-1} \mid S \in \mathrm{Sp}(2 n, \mathbb{R})\right\} .
$$

Then each $\underline{J}(\underline{U}, \underline{V})$ belongs to $\mathcal{O}$, and so does $J^{\prime}$ obtained from $\underline{J}(\underline{U}, \underline{V})$ by Eq. (3.3). What must be shown, in order to clinch the issue, is that each point on $\mathcal{O}$ is uniquely characterized by a pair of real symmetric $\mathrm{n} \times n$ matrices $(\underline{U}, \underline{V})$ with $\underline{U}$ positive definite; or in other words, that such $(\underline{U}, \underline{V})$ give a global coordinate system for $\mathcal{O}$. If this is so, then Eq. (2.29) and the corresponding $J$ equation would follow

$$
\begin{aligned}
& \left(\underline{S}^{-1}\right)^{T} \underline{G}(\underline{U}, \underline{V}) \underline{S} \underline{S}^{-1}=\underline{G}\left(\underline{U}^{\prime}, \underline{V}^{\prime}\right), \\
& \underline{S} J(\underline{U}, \underline{V}) \underline{S}^{-1}=\underline{J}\left(\underline{U}^{\prime}, \underline{V}^{\prime}\right) .
\end{aligned}
$$

Before doing this, however, we note two interesting consequences of the already established Eq. (3.4). The first is that we have here an explicit and simple instance of a fundamental theorem of Williamson" which states that any real symmetric positive-definite $2 \mathrm{n} \times 2 \mathrm{n}$ matrix $\mathrm{G}$ can be brought to diagonal form, with positive diagonal entries, by a symmetric transformation with a symplectic matrix $S \in \operatorname{Sp}(2 n, \mathbb{R})$. The diagonal matrix concerned is called the normal form of $\underline{G}$. In this sense, the normal form of each $\underline{G}(\underline{U}, \underline{V})$ is the unit matrix. Incidentally this means that any two such matrices, $\underline{G}(\underline{\underline{U}}, \underline{V})$ and $\underline{G}\left(\underline{U}^{\prime}, \underline{V}^{\prime}\right)$ are related by a suitable symplectic transformation. The second point is that any GPS $\left|\psi_{Z}\right\rangle$ can be unitarily related via $\mathcal{U}(S)$ for a suitable $S \in \mathrm{Sp}(2 \mathrm{n}, \mathbb{R})$ to the standard GPS $\left|\psi_{Z^{(0)}}\right\rangle$, where $\underline{U}^{(0)}=\underline{\mathbb{1}}_{n \times n}$ and $\underline{V}^{(0)}$ $=0, x_{n}$, up to a phase factor,

$$
\begin{aligned}
& \left|\psi_{Z}\right\rangle \doteq \mathcal{U}(S(U, V))\left|\psi_{Z^{(0)}}\right\rangle \\
& \psi_{Z^{(0)}}(q)=\pi^{-n / 4} \exp \left(-\frac{1}{2} \underline{q}^{T} \underline{q}\right)
\end{aligned}
$$

We now prove our main result concerning the possibility of using $(\underline{U}, \underline{V})$ as a global coordinate system for $\mathcal{O}$. We want to show that for any $J \in \mathcal{O}, \underline{G}=-\underline{\beta} \underline{J}$ is $\underline{G}(\underline{U}, \underline{V})$ for a unique pair $(\underline{U}, \underline{V})$. Starting with the Williamson normal form $\underline{G}^{(0)}=\underline{1}_{2 n \times 2 n}$, we consider the family of $\underline{G}$ matrices 


$$
\widetilde{\mathcal{O}}=\left\{\left(\underline{S}^{-1}\right)^{T} \underline{S}^{-1} \mid S \in \mathrm{Sp}(2 n, \mathbb{R})\right\} .
$$

This set of matrices is just $-\underline{\beta}$ times the set of generator matrices $\mathcal{O}$ : We have passed from the $\underline{J}$ to the $\underline{G}$ description. Every $\underline{G} \in \mathbf{\sigma}$ has the following three properties:

$G$ is real symmetric,

$\underline{G}$ is positive definite ,

$G \in \operatorname{Sp}(2 n, \mathrm{R})$.

Conversely one can easily check using Williamson's theorem that any $\underline{G}$ having these properties belongs to $\widetilde{O}$. So we have

$$
\widetilde{\mathcal{O}}=\{\underline{G} \mid \underline{G} \text { obeys Eqs. (3.9) }\} .
$$

In this form we can easily show that any $\underline{G} \in \widetilde{\mathcal{O}}$ is $\underline{G}(\underline{U}, \underline{V})$ as given in Eq. (2.27), for a unique pair $(\underline{U}, \underline{V})$. Properties (3.9a) and (3.9b) imply that if $\underline{G}$ is written as in Eq. (2.19) with $n \times n$ real submatrices $\underline{A}, \underline{B}$, and $\underline{C}$, then $\underline{A}$ and $\underline{B}$ are symmetric positive definite:

$$
\underline{G}=\left[\begin{array}{ll}
\underline{A} & \underline{C} \\
\underline{C}^{T} & \underline{B}
\end{array}\right], \quad \underline{A}^{T}=\underline{A}>\underline{0}, \quad \underline{B}^{T}=\underline{B}>\underline{0} .
$$

(The positive definiteness of $\underline{G}$ implies more than this.) We now impose the symplectic condition (2.4) on $\underline{G}$ to get three independent matrix relations:

$$
\begin{aligned}
& \underline{C}^{T} \underline{B}=\underline{B C}, \\
& \underline{A B}=\underline{1}_{n \times n}+\underline{C}^{2},
\end{aligned}
$$

$$
\underline{A C}^{T}=\underline{C A} .
$$

Keeping in view the form of $\underline{G}(\underline{U}, \underline{V})$ in Eq. (2.27), we write

$$
\underline{B}=\underline{U}^{-1}, \quad \underline{C}=\underline{V U}^{-1},
$$

thereby defining uniquely a real symmetric positive definite $\underline{U}$ and a real $\underline{V}$. Then Eqs. (3.12) are, in the same sequence,

$$
\begin{aligned}
& \underline{U}^{-1} \underline{V}^{T} \underline{U}^{-1}=\underline{U}^{-1} \underline{V U}^{-1}, \\
& \underline{A}=\underline{U}+\underline{V U}{ }^{-1} \underline{V}, \\
& \underline{A U}{ }^{-1} \underline{V}^{T}=\underline{V U}^{-1} \underline{A} .
\end{aligned}
$$

We get from Eq. (3.14a) the symmetry of $\underline{V}$; then (3.14b) gives $\underline{A}$ explicitly in terms of the pair $(\underline{U}, \underline{V})$ while $(3.14 \mathrm{c})$ is identically satisfied. Thus the $\underline{G}$ of Eq. (3.11) has been shown to be $\underline{G}(\underline{U}, \underline{V})$ for the unique pair $(\underline{U}, \underline{V})$ identified by Eq. (3.13). At the same time, the positive definiteness of $\underline{G}(\underline{U}, \underline{V})$ is obvious from its factored form-in Eq. (2.27).

It is thus established that each $G \in \mathcal{O} \quad(J \in \mathcal{O})$ is $\underline{\underline{G}}(\underline{U}, \underline{V})[\underline{J}(\underline{U}, \underline{V})]$ for a unique $(\underline{U}, \underline{V})$ and vice versa; therefore Eqs. (3.6) do hold with $\left(\underline{U}^{\prime}, \underline{V}^{\prime}\right)$ being determined as functions of $(\underline{U}, \underline{V})$ and $\mathbf{S .} \mathrm{We}$ conclude this section by developing an explicit solution for $\left(\underline{U}^{\prime}, \underline{V}^{\prime}\right)$.

We use the factorization of $\underline{G}(\underline{U}, \underline{V})$ in terms of $\underline{S}(\underline{U}, \underline{V})$ given in Eq. (3.2) [similarly for $\underline{G}\left(\underline{U}^{\prime}, \underline{V}^{\prime}\right)$ ] and exploit the symplectic property (2.4) of these matrices and of $\underline{S}$, to express Eq. (3.6)in this way:

$$
\begin{aligned}
& \underline{G}\left(\underline{U}^{\prime}, \underline{V}^{\prime}\right)=\left(\underline{S}^{-1}\right)^{T} \underline{G}(\underline{U}, \underline{V}) \underline{S} \underline{-1}^{-1}-\left[\underline{S}\left(\underline{U}^{\prime}, \underline{V}^{\prime}\right)^{-1}\right]^{T}\left(\underline{1}_{2 n \times 2 n}-i \underline{\beta}\right) \underline{S}^{-1}\left(\underline{U}^{\prime}, \underline{V}^{\prime}\right) \\
& =\left(\underline{S}^{-1}\right)^{T}\left[\underline{S}(\underline{U}, \underline{V})^{-1}\right]^{T}\left(\underline{\mathbb{1}}_{2 n \times 2 n}-i \underline{\beta}\right) S^{-1}(\underline{U}, \underline{V}) \underline{S}^{-1}-\left[\underline{S}^{-1}\left(\underline{U}^{\prime}, \underline{V}^{\prime}\right)\right]^{T}\left[\begin{array}{c}
\underline{\mathbb{1}}_{n \times n} \\
i \underline{1}_{n \times n}
\end{array}\right)\left(\underline{\mathbb{1}}_{n \times n}-i \underline{\mathbb{1}}_{n \times n}\right) \underline{S}^{-1}\left(\underline{U}^{\prime}, \underline{V}^{\prime}\right) \\
& =\left(\underline{\boldsymbol{S}}^{-1}\right)^{T}\left[\underline{\boldsymbol{S}}(\underline{\boldsymbol{U}}, \underline{\boldsymbol{V}})^{-1}\right]^{T}\left[\begin{array}{c}
\underline{\mathbf{1}}_{n \times n} \\
i \mathbb{1}_{n \times n}
\end{array}\right]\left(\underline{\mathbf{1}}_{n \times n}-i \underline{\mathbb{1}}_{n \times n}\right) \underline{\boldsymbol{S}}^{-1}(\underline{\boldsymbol{U}}, \underline{\boldsymbol{V}}) \underline{\boldsymbol{S}}^{-1}
\end{aligned}
$$

This motivates the definition

$\underline{\xi}(\underline{U}, \underline{V})=\left[\underline{S}(\underline{U}, \underline{V})^{-1}\right]^{T}\left(\begin{array}{c}\underline{1}_{n \times n} \\ i \underline{\underline{1}}_{n \times n}\end{array}\right)=i\left(\begin{array}{c}\underline{\boldsymbol{Z}} \\ \underline{\underline{1}}_{n \times n}\end{array}\right) \underline{U}^{-1 / 2}$,

where $\underline{Z}=\underline{V}-i \underline{U}$ as before. Therefore Eq. (3.15), which must yield $\underline{\underline{Z}}$ ' in terms of $\underline{\boldsymbol{Z}}$ and $\underline{\underline{S}}$, is

$$
\xi\left(\underline{U}^{\prime}, \underline{V}^{\prime}\right) \xi^{+}\left(\underline{U}^{\prime}, \underline{V}^{\prime}\right)=\left(\underline{S}^{-1}\right)^{\top} \underline{\xi}(\underline{U}, \underline{V}) \underline{\xi}(\underline{U}, \underline{V}) \underline{S}^{-1} .
$$

The strategy is to extract $\underline{\xi}\left(\underline{U}^{\prime}, \underline{V}^{\prime}\right)$ in terms of $\underline{\xi}(\underline{U}, \underline{V})$ and $\underline{S}$. For ease in the ensuing manipulations, let us write the $2 \mathrm{n} \times n$ matrices

$$
\underline{X}=\left(\underline{S}^{-1}\right)^{T} \xi(\underline{U}, \underline{V}),
$$

and

$$
\underline{Y}=\underline{\xi}\left(\underline{U}^{\prime}, \underline{V}^{\prime}\right),
$$

so Eq. (3.17) is

$$
\underline{Y Y}^{+}=\underline{X X}^{\dagger} .
$$

Now

$$
\begin{aligned}
\underline{Y}^{\dagger} \underline{Y} & =\xi^{\dagger}\left(\underline{U}^{\prime}, \underline{V}^{\prime}\right) \xi\left(\underline{U}^{\prime}, \underline{V}^{\prime}\right) \\
& =\left(\underline{U}^{\prime}\right)-{ }^{1 / 2}\left[\underline{\mathbb{1}}_{n \times n}+\left(\underline{Z}^{\prime}\right)^{\dagger} \underline{Z}^{\prime}\right]\left(\underline{U}^{\prime}\right)-{ }^{1 / 2}
\end{aligned}
$$

is evidently Hermitian, positive definite, and nonsingular. From Eq. (3.19)we get

$$
\left(\underline{Y}^{\mathrm{t}} \underline{Y}\right)^{2}=(\underline{X}+\underline{Y}) \mathrm{t}(\underline{X}+\underline{Y}),
$$

proving that $\underline{X}^{\dagger} \underline{Y}$ is nonsingular. We also get from Eq. (3.19)

$$
\underline{\boldsymbol{Y}}{ }^{\dagger} \underline{\boldsymbol{Y}}=\underline{\boldsymbol{X X}}_{-}^{\dagger} \underline{\boldsymbol{Y}},
$$

which allows us to "solve" for $\underline{Y}$ in terms of $\underline{X}$ : 


$$
\begin{aligned}
& \underline{Y}=\underline{X W} \\
& \underline{W}=\underline{X}^{\dagger} \underline{Y}\left(\underline{Y}^{\dagger} \underline{Y}\right)^{-1}
\end{aligned}
$$

The important property of the $\mathrm{n} \times n$ matrix $\underline{W}$ is that it is unitary, as shown by Eq. (3.21):

$$
\underline{W}^{\dagger} \underline{W}^{\prime} \underline{\mathbb{1}}_{n \times n} .
$$

If we write the symplectic matrix $\underline{S}$ and its inverse in block form as

$$
\underline{S}=\left|\begin{array}{l}
\underline{b} \\
\underline{d}
\end{array}\right|, \quad \underline{S}^{-1}=\left|\begin{array}{cc}
\underline{d}^{T} & -\underline{b}^{T} \\
-\underline{c}^{T} & \underline{\underline{a}}^{T}
\end{array}\right|,
$$

then Eq. (3.23)gives the $n \times n$ matrix equations

$$
\begin{aligned}
& \underline{Z}^{\prime}\left(\underline{U}^{\prime}\right)^{-1 / 2}=(\underline{d Z}-\underline{c}) \underline{U}-1 / 2 \underline{W}, \\
& \left(\underline{U}^{\prime}\right)^{-1 / 2}=(\underline{a}-\underline{b Z}) \underline{U}{ }^{-1 / 2} \underline{W} .
\end{aligned}
$$

The existence of an inverse to $(\underline{a}-\underline{b Z})$ is assured since we know that $\underline{U}, \underline{U}^{\prime}$, and $\underline{W}$ are all nonsingular. Therefore, even though $\underline{W}$ containing the "unknown" $\underline{Y}$ appears on the right-hand sides of these two equations, we can combine them to give us the solution for $\underline{Z}^{\prime}$ in terms of $\underline{\boldsymbol{Z}}$ and $\underline{\boldsymbol{S}}$ that we are looking for,

$$
\underline{Z}^{\prime}=(\underline{d Z}-\underline{c})(\underline{a}-\underline{b Z})^{-1} \text {. }
$$

This is the transformation law for $Z$ under $\operatorname{Sp}(2 n, \mathbb{R})$ promised earlier. $\underline{U}^{\prime}$ and $\underline{V}^{\prime}$ can be obtained by separating $\underline{Z}^{\prime}$ into its imaginary and real parts, remembering that $\underline{\underline{a}}, \underline{b}, \underline{c}$, and $\underline{\boldsymbol{d}}$ are all real. Thus Eq. (3.27) expresses the (nonlinear) adjoint action of $\operatorname{Sp}(2 n, \mathbb{R})$ on the orbit $\mathbf{O}$ (equally well $\mathscr{O}$ ), telling us how an element $S \in \operatorname{Sp}(2 n, \mathbb{R})$ maps a point $(\underline{U}, \underline{V})$ on $\mathbf{O}$ to $\left(\underline{U}^{\prime}, \underline{V}^{\prime}\right)$. For future reference, we rewrite Eq. (3.27) in terms of $\underline{\Lambda} \equiv-\underline{Z}^{-}$(the positive definiteness of $\underline{U}$ guarantees that $\underline{\boldsymbol{Z}}$ is nonsingular):

$$
\underline{\Lambda}^{\prime}=(\underline{a} \underline{\Lambda}+\underline{b})(\underline{c} \underline{\Lambda}+\underline{d})^{-1} .
$$

Going back to the GPS's (with $Q_{0}=0$ ), we can complete Eqs. (2.28) to the statements

$$
\begin{aligned}
& \mathcal{U}(S) \hat{\rho}_{Z} \mathcal{U}^{-1}(S)=\hat{\rho}_{Z^{\prime}}, \\
& W_{Z}\left(S^{-1} Q\right)=W_{Z^{\prime}}(Q),
\end{aligned}
$$

which establishes and shows exactly how each such state is taken into another such state by any element of $S p(2 n, \mathbb{R})$. Reinstating the displacement $Q_{0}$ in the GPS's is a trivial matter because of the semidirect product structure noted earlier, and need not be spelled out in detail.

\section{SCHRÖDINGER EQUATION FOR GPS's AS AN EQUIVALENT CLASSICAL SYSTEM}

We have seen how a GPS $\left|\psi_{Z}\right\rangle$ changes under action by $\mathcal{U}(S)$ into another GPS $\left|\psi_{Z^{\prime}}\right\rangle$, with $S$ being any element of $S p(2 n, \mathbb{R})$. We now specialize to the solution of the Schrodinger equation for a given quadratic Hamiltonian.
Let the Hamiltonian operator $\hat{H}$ be determined by a real symmetric $2 \mathrm{n} \times 2 n$ matrix $\underline{h}$, and let us use Eq. (2.9) to write it as one of the generators of $\mathcal{U}(S)$,

$$
\begin{aligned}
& \hat{H}=\frac{1}{2} \hat{Q}^{T} h \hat{Q}=X\left(J_{0}\right), \\
& J_{0}=\beta h .
\end{aligned}
$$

The unitary time-evolution operator for a finite time $t$ is

$$
\begin{aligned}
& e^{-i t \hat{A}}=e^{-i t X\left(J_{0}\right)}=U(S(t)), \\
& S(t)=e^{t J_{0}} \in \operatorname{Sp}(2 n, \mathbb{R}) .
\end{aligned}
$$

Here we used the general connection

$$
\mathcal{U}\left(e^{\epsilon J}\right)=e^{-i \epsilon X(J)}
$$

implied by Eqs. (2.7) and (2.9). Therefore if at time $t=0$ we have a GPS $\left|\psi_{\boldsymbol{Z}(0)}\right\rangle$ which evolves to $\left|\psi_{\boldsymbol{Z}(t)}\right\rangle$ at time $t$,

$$
\begin{aligned}
\left|\psi_{Z(t)}\right\rangle & =e^{-i t \hat{H}}\left|\psi_{Z(0)}\right\rangle \\
& =\mathcal{U}(S(t))\left|\psi_{Z(0)}\right\rangle,
\end{aligned}
$$

then the matrix $\underline{G}(0)$ for the initial Wigner-Moyal distribution evolves to $\underline{G}(t)$ by

$$
\underline{G}(t)=\left[\underline{S}^{-1}(t)\right]^{T} \underline{G}(0) \underline{S}^{-1}(t) .
$$

This is obviously the solution to the linear differential equation

$$
\begin{aligned}
\frac{d \underline{G}(t)}{d t} & =-\underline{J}_{0}^{T} \underline{G}(t)-\underline{G}(t) \underline{J}_{0} \\
& =\underline{\beta}_{0} \underline{\beta}^{-1} \underline{G}(t)-\underline{G}(t) \underline{J}_{0}
\end{aligned}
$$

with prescribed initial conditions. In fact, of course, $\underline{G}(t)$ isdetermined by $\underline{Z}(t)=\underline{V}(t)-i \underline{U}(t)$ as

$$
G(t)=\underline{G}(\underline{U}(t), \underline{V}(t))
$$

in the notation of Eq. (2.27); and the primitive evolution equations, of which (4.6) must be viewed as a consequence, are nonlinear ordinary differential equations for $\underline{U}(t), \underline{V}(t)$. The essential point is that the quantummechanical Schrodinger equation for the GPS $\left|\psi_{\boldsymbol{Z}(t)}\right\rangle$ reduces to these evolution equations for $\boldsymbol{Z}(t)$ or $G(t)$. It now turns out that these latter equations can be put into a classical canonical form in an intrinsic and natural way, on account of the geometrical properties of the orbit $O \subset \operatorname{sp}(2 n, \mathbb{R})$. We show this by first exposing the nature of $\mathrm{O}$ as a coset space, and then appealing to general theorems which allow the setting up of a classical phasespace structure on $\mathbf{O}$.

From the definition of $\mathbf{O}$ in Eq. (3.5), it is clear that the action of $\mathrm{Sp}(2 n, \mathbb{R})$ on $O$ is transitive. This means that $\mathcal{O}$ is essentially the coset space $\operatorname{Sp}(2 n, \mathbb{R}) / H$, where $H$ is the subgroup of $\operatorname{Sp}(2 n, \mathbb{R})$ which leaves the representative point $\underline{J}^{(0)}=\beta$ on $\mathcal{O}$ fixed. Since the dimensions of $\operatorname{Sp}(2 n, \mathbb{R})$ and $\mathrm{O}$ are $n(2 n+1)$ and $n(n+1)$, respectively [the latter follows from the fact that $(\underline{U}, \underline{V})$ is a coordinate system for $\mathcal{O}$ ], $\boldsymbol{H}$ must be an $n^{2}$-dimensional subgroup. The elements $\mathrm{S}$ in $H$ must obey the two conditions 


$$
\begin{aligned}
& S^{T} \beta S=\beta, \\
& S^{-1} \beta S=\beta,
\end{aligned}
$$

which means $\underline{S}^{T}=\underline{S}^{-1}$ or $S \in \mathrm{SO}(2 n)$. Therefore $\mathrm{H}$ is the intersection

$$
H=\operatorname{Sp}(2 n, \mathbb{R}) \cap \operatorname{SO}(2 n) .
$$

By examining the infinitesimal generators of $H$, we shall show that $H=\mathrm{U}(n)$. For $\underline{J} \in \mathcal{H}$, the Lie algebra of $\boldsymbol{H}$, the two conditions (4.8) lead to

$$
\begin{aligned}
& (\underline{\beta} \underline{J})^{T}=\underline{\beta} \underline{J}, \\
& \underline{J}^{T}=-\underline{J} .
\end{aligned}
$$

Every $\underline{J} \in \mathcal{H}$ is thus a real $2 \mathrm{n} \times 2 n$ matrix whose block form is

$$
\begin{aligned}
& \underline{J}=\left(\begin{array}{cc}
\underline{\lambda} & -\underline{\mu} \\
\underline{\mu} & \underline{\lambda}
\end{array}\right), \\
& \underline{\lambda}^{T}=-\underline{\lambda}, \underline{\mu}^{T}=\underline{\mu} .
\end{aligned}
$$

Here $\underline{\lambda}$ and $\underline{\mu}$ are real $\mathrm{n} \times n$ matrices. The infinitesimal transformation (2.7) acting on a column vector $Q$ produces the changes

$$
\begin{aligned}
& q^{\prime} \simeq q+\epsilon(\lambda q-\mu p), \\
& p^{\prime} \simeq p+\epsilon(\lambda p+y q) .
\end{aligned}
$$

These real equations are identical to the single complex matrix transformation equation

$$
\underline{q}^{\prime}+i \underline{p}^{\prime} \simeq\left[\underline{1}_{n \times n}+\epsilon(\underline{\lambda}+i \underline{\mu})\right](\underline{q}+i \underline{p}) .
$$

Since $\underline{\lambda}+i \underline{\mu}$ is the most general anti-Hermitian $n \times n$ matrix, we have here a general infinitesimal transformation of the unitary group $U(n)$. This establishes that

$$
\begin{aligned}
& H=\mathrm{Sp}(2 n, \mathbb{R}) \cap \mathrm{SO}(2 n)=\mathrm{U}(n), \\
& O=\mathrm{Sp}(2 n, \mathbb{R}) / \mathrm{U}(n) .
\end{aligned}
$$

We now want to give the evolution equations (4.6) a classical canonical meaning. These equations describe a particular one-parameter group of motions along the orbit $\mathcal{O}$. According to a general theorem due to Kostant, Kirillov, and Souriau, ${ }^{12}$ for any Lie group $G$ the coset spaces $\mathrm{G} / H$ which permit the definition of a G-invariant symplectic structure are either orbits in the coadjoint representation of $\mathrm{G}$ acting on $\mathcal{G}^{*}$ (the dual to the Lie algebra 9 of $G$ ), or covering spaces of such orbits. For a semisimple G, such as $\mathbf{S p}(2 n, \mathbb{R})$, we can deal with orbits in $S$ rather than in $S^{*}$; and every orbit in 9 does carry a G-invariant symplectic structure. This is in particular true for $G=\mathrm{Sp}(2 n, \mathbb{R})$ and the orbit $O \subset \operatorname{sp}(2 n, \mathbb{R})$. In principle the symplectic structure can be defined directly and intrinsically on $\mathcal{O}$. However, both for calculational ease and physical understanding it is better to define a singular system of generalized Poisson brackets on the full Lie algebra $\operatorname{sp}(2 n, \mathbb{R})$, and then restrict them to the orbit $\mathcal{O}$. The $\operatorname{Sp}(2 n, \mathbb{R})$ invariance (better, covariance) of the procedure will be obvious throughout.

We need to set up the commutation relations for $\operatorname{sp}(2 n, \mathbb{R})$ in a convenient way. In the defining representation, a basis $X_{a b}$ for $\operatorname{sp}(2 n, \mathbb{R})$ is obtained by taking a basis $Y_{a b}$ for real symmetric $2 \mathrm{n} \times 2 n$ matrices, and multiplying them on the left by $\beta$. [This is the convention used in Eqs. (3.1), (3.3), and (4.1).] We make the choice

$$
\begin{aligned}
& Y_{a b}=Y_{b a}, \quad a, b=1,2, \ldots, 2 n \\
& \left(Y_{a b}\right)_{c d}=\delta_{a c} \delta_{b d}+\delta_{a d} \delta_{b c}
\end{aligned}
$$

so that a general real symmetric $2 \mathrm{n} \times 2 \mathrm{n}$ matrix $\underline{G}=\left(G_{a b}\right)$ has the expansion

$$
G=\frac{1}{2} G_{a b} Y_{a b} .
$$

A basis for $\operatorname{sp}(2 n, \mathbb{R})$ is then

$$
\begin{aligned}
& X_{a b}=X_{b a}=\beta Y_{a b}, \\
& \left(X_{a b}\right)_{c d}=\beta_{c a} \delta_{b d}+\beta_{c b} \delta_{a d} .
\end{aligned}
$$

The commutation relations among the $X_{a b}$ are calculated to be

$\left[X_{a b}, X_{c d}\right]=\beta_{a c} X_{b d}+\beta_{b c} X_{a d}+\beta_{a d} X_{c b}+\beta_{b d} X_{c a}$.

These are the basic Lie bracket relations for $\operatorname{sp}(2 n, \mathbb{R})$. The generator matrix $\underline{J}=\underline{B} \underline{G}$ has the expansion

$$
J=\frac{1}{2} G_{\mathrm{ab}} X_{\mathrm{ah}}
$$

to accompany (4.16), so the symmetric coefficients $G_{a b}$ are to be treated as the independent components of $\mathbf{J} \in \operatorname{sp}(2 n, \mathbb{R})$. That is, the $n(2 \mathrm{n}+1)$ independent real variables $G_{a b}=G_{b a}$ are (linear) coordinates for $\operatorname{sp}(2 n, \mathbb{R})$. Among them we define a system of singular generalized Poisson brackets patterned after Eq. (4.18),

$\left\{G_{a b}, G_{c d}\right\}=\beta_{a c} G_{b d}+\beta_{b c} G_{a d}+\beta_{a d} G_{c b}+\beta_{b d} G_{c a}$.

These are manifestly covariant under the action of $\operatorname{Sp}(2 n, \mathbb{R})$ on $G_{a b}$, which is given by

$$
G_{a b} \rightarrow G_{a b}^{\prime}=\left(\underline{S}^{-1}\right)_{c a}\left(\underline{S}^{-1}\right)_{d b} G_{c d} .
$$

The Poisson bracket between any two functions $f(G)$, $g(\mathrm{G})$ is calculated from the basic brackets (4.20) by using the derivation property.

At this stage, one can easily see that the (linear)evolution equations (4.6) can be put into the classical canonical form, in the sense of the Poisson bracket (4.20), with the use of a Hamiltonian function linear in the G's,

$$
\frac{d G_{a b}}{d t}=\left\{G_{a b},-\frac{1}{2}(\underline{\beta} \underline{h} \underline{\beta})_{c d} G_{c d}\right\} .
$$

However, as already remarked, the primitive evolution equations, corresponding to the quantum-mechanical equation of motion for a GPS, are not really Eq. (4.6) but the nonlinear evolution equations for $U(t)$ and $V(t)$ implied by Eq. (4.6). This aspect is closely related to the question of restricting the Poisson bracket (4.20) to (the orbit) $\widetilde{\mathcal{O}}$, a procedure which can be consistently carried out because (4.20) is $\mathbf{S p}(2 n, \mathbb{R})$ covariant. Namely, if $\mathcal{C}(\mathrm{G})$ is any (polynomial) Casimir invariant of $\operatorname{sp}(2 n, \mathbb{R})$, the singularity of the brackets (4.20) shows up in the fact that 


$$
\left\{G_{a b}, \mathcal{Q}(G)\right\}=0
$$

identically. Therefore we are permitted to assign each $\mathcal{C}(G)$ some numerical value, without in any way conflicting with the Poisson-bracket definition (4.20). The independent $\mathcal{C}(\boldsymbol{G})$ are the traces of even powers of $\underline{\beta} \underline{G}$, and the restriction of $\underline{G}$ to $\widetilde{\mathcal{O}}$ is achieved by specifying

$$
\operatorname{Tr}\left[(\beta \underline{G})^{2 l}\right]=(-1)^{l} \times 2 n, \quad l=1,2, \ldots, n .
$$

Once we know that $G \in \widetilde{\mathcal{O}}$, we can use the variables $(\underline{U}, \underline{V})$ as independent coordinates on $\widetilde{\mathcal{O}}$; the brackets (4.20) then imply definite values for the brackets among the elements of $\underline{U}$ and $\underline{V}$. In this way we find that (4.20) leads to the canonical Poisson brackets ${ }^{2}$

$$
\begin{aligned}
& \left\{V_{r s},\left(\underline{U}^{-1}\right)_{r^{\prime} s^{\prime}}\right\}=\delta_{r r^{\prime}} \delta_{s s^{\prime}}+\delta_{r s^{\prime}} \delta_{s r^{\prime}}, \\
& \left\{V_{r s}, V_{r^{\prime} s^{\prime}}\right\}=\left\{\left(\underline{U}^{-1}\right)_{r s},\left(\underline{U}^{-1}\right)_{r^{\prime} s^{\prime}}\right\}=0 .
\end{aligned}
$$

Thus the $\underline{V}^{\prime} s$ and $\underline{U}^{-1}$ 's can be thought of as q's and $p$ 's intrinsically and globally defined on $\widetilde{\mathcal{O}}$ (or $\mathcal{O}$ ). And the primitive equations of motion for $U(t)$ and $V(t)$, describing the unitary time development of GPS's under the Hamiltonian operator $\hat{H}$ of Eq. (4.1), are

$$
\begin{aligned}
& \left.\frac{d \underline{U}}{d t}=I L L,-\frac{1}{2}(\underline{\beta} \underline{\underline{B}})_{a b}[\underline{G}(\underline{U}, \underline{V})]_{a b}\right\}, \\
& \frac{d \underline{V}}{d t}=\left\{V,-\frac{1}{2}(\underline{\beta} \underline{\underline{\beta}})_{a b}[\underline{G}(\underline{U}, \underline{V})]_{a b}\right\} .
\end{aligned}
$$

The nonlinear nature of these equations is evident when one sees how $\underline{G}(\underline{U}, \underline{V})$ is built up from $(\underline{U}, \underline{V})$ in Eq. (2.27). However, the solution is already known to us [a particular case of Eq. (3.27)], and is a canonical transformation on 6 . We conclude this section by noting that the particular Hamiltonian studied by Birula ${ }^{2}$ corresponds to

$$
\boldsymbol{h}=\left(\begin{array}{ll}
\underline{\Omega}^{2} & \underline{0}_{n \times n} \\
\underline{0}_{n \times n} & \underline{1}_{n \times n}
\end{array}\right),
$$

and in that case Eq. (4.26)above gives his Eq. (2.19).

\section{APPLICATIONTO GAUSSIAN SCHELL-MODEL FIELDS IN OPTICS}

The results on the action of $\operatorname{Sp}(2 n, \mathbb{R})$ on GPS's find an interesting application, in the case $n=2$, to a special class of partially coherent optical fields contained among the so-called anisotropic Gaussian Schell-model (AGSM) fields. ${ }^{13}$ Both isotropic Gaussian Schell-model fields ${ }^{14,15}$ and AGSM fields ${ }^{16}$ have played a key role in recent studies in radiometry of partially coherent sources. Like other paraxial optical fields, the two-point correlation function" of such a field can be specified on a twodimensional plane transverse to the beam axis, and it then evolves along the axis. A general AGSM field has a two-point function (cross-spectral density) $\Gamma_{L, M, K}\left(q ; q^{\prime}\right)$ of the form given in Eq. (2.18), except for an overall real multiplicative constant. (A dependence on the frequency $\omega$ has for brevity been omitted.) It is characterized by the three real $2 \times 2$ matrices $\underline{L}, \underline{M}$, and $\underline{K}$. Apart from the requirements that $\underline{L}$ and $\underline{L}+\underline{M}$ be positive definite, there are two additional conditions (involving $\underline{\underline{L}}, \underline{M}$, and the antisymmetric part of $\underline{K})^{5}$ which must be satisfied if $\hat{\Gamma}_{L, M, K}$ is to be a positive-semidefinite operator, which is necessary for $\Gamma_{L, M, K}\left(q ; q^{\prime}\right)$ to be an acceptable optical twopoint function.

First-order optical systems (FOS's), ${ }^{18}$ of which free paraxial propagation is an example, act in an especially simple way on paraxial fields. Each such system can be represented as an element $S$ of $S p(4, \mathbb{R})$. Elsewhere we have shown that every $S \in \operatorname{Sp}(4, \mathbb{R})$, including inverse free propagation, can be synthesized using thin lenses." Its action on the so-called Wolf function ${ }^{20} W(\mathrm{Q})$ [related to the optical two-point function $\Gamma\left(q ; q^{\prime}\right)$ by Eq. (2.13)] is given by Eq. (2.14). It follows that if we have an AGSM field $\Gamma_{L, M, K}\left(q ; q^{\prime}\right)$, its Wolf function is Gaussian and is characterized by a $4 \times 4$ matrix $\underline{G}$ built up from $\underline{L}, \underline{M}, \underline{K}$ as in Eq. (2.19); and when this field passes through an FOS corresponding to $S \in S p(4, \mathbb{R})$, we get an altered AGSM field whose matrix $\underline{G}^{\prime}$ is

$$
\underline{\underline{S}}^{\prime}=\left(\underline{S}^{-1}\right)^{T} \underline{G S}{ }^{-1} \text {. }
$$

Let us now consider all those AGSM fields whose $\underline{L}$, $\underline{M}$, and $\underline{K}$ are such that the Williamson normal form of $\underline{G}$ is a multiple of the $4 \times 4$ unit matrix

$$
\underline{G}^{(0)}=\kappa \underline{1}_{4 \times 4}, \quad 0<\kappa \leq 1
$$

The limits on $\boldsymbol{k}$ arise from the extra conditions mentioned above that ensure that $\hat{\Gamma}_{L, M, K}$ is positive semidefinite. Then $\underline{G}$ must be of the form

$$
G=\kappa\left(\underline{S}^{-1}\right)^{T} \underline{S}^{-1}, \quad S \in \operatorname{Sp}(4, \mathbb{R}) .
$$

It follows that each such $\underline{G}$ is $\boldsymbol{k}$ times some element of the set of matrices $\widetilde{\mathcal{O}}$ defined in Eqs. (3.8) and (3.10) (for $n=2)$, so there must be a unique pair $(\underline{U}, \underline{V})$ such that

$$
\underline{G}=\kappa \underline{G}(\underline{U}, \underline{V}) \text {. }
$$

This collection of matrices can be denoted $\widetilde{O}_{\kappa}$ : The previous $\mathbf{6}$ is $\mathbf{6}$, and corresponds, in the present optical context, to coherent anisotropic Gaussian beams. ${ }^{21}$ Then, for each $\kappa,(\underline{U}, \underline{V})$ forms a global coordinate system for $6 ;$ and 6 , is a realization of the coset space $\mathrm{Sp}(4, \mathbb{R}) / \mathrm{U}(2)$. We can invert Eqs. (2.19) to get $\underline{\underline{L}}, \underline{M}$, and $\underline{K}$ for an AGSM field whose $\underline{G}$ matrix is $\kappa \underline{G}(\underline{U}, \underline{\underline{V}})$,

$$
\begin{aligned}
& \underline{L}=\frac{\kappa}{2} \underline{U}, \\
& \underline{M}=\frac{1-\kappa^{2}}{2 \kappa} \underline{U}, \\
& \underline{K}=-\underline{V} .
\end{aligned}
$$

As a subset of the set of all AGSM fields, what we have here is what was called Type I in Ref. 13. An $S p(4, R)$. invariant characterization of the $\underline{G}$ matrices of such fields is

$$
\begin{aligned}
& \operatorname{Tr}\left[(\underline{\beta} \underline{G})^{2}\right]=-4 \kappa^{2}, \\
& \operatorname{Tr}\left[(\underline{\beta} \underline{G})^{4}\right]=4 \kappa^{4}, \quad 0<\kappa \leq 1
\end{aligned}
$$


We see from Eqs. (3.6) and (5.4)that under action by the FOS $S \in \mathbf{S p}(4, \mathbb{R})$, the $\mathbf{G}$ matrix changes thus:

$\underline{G}=\kappa \underline{G}(\underline{U}, \underline{V}) \rightarrow \underline{G}^{\prime}=\left(\underline{S}^{-1}\right)^{T} \underline{G} \underline{S}{ }^{-1}=\kappa \underline{G}\left(\underline{U}^{\prime}, \underline{V}^{\prime}\right)$.

The rule for calculating $\left(\underline{U}^{\prime}, \underline{V}^{\prime}\right)$ from $(\underline{U}, \underline{V})$ and $\underline{S}$ is the same as with GPS's, i.e., the "abcd law" remains valid,

$$
\begin{aligned}
& \kappa^{\prime}=\kappa, \\
& \underline{Z}^{\prime}=\underline{V}^{\prime}-i \underline{U^{\prime}}=(\underline{d Z}-\underline{c})(\underline{a}-\underline{b Z})^{-1}, \\
& \underline{\Lambda}^{\prime}=(\underline{a} \underline{\Lambda}+\underline{b})(\underline{c} \underline{\Lambda}+\underline{d})^{-1},
\end{aligned}
$$

where $\underline{a}, \underline{b}, \underline{c}$, and $\underline{d}$ are $2 \times 2$ blocks making up $\underline{S}$. We also see from Eq. (5.8) that the matrix $\underline{K}$, determining the phase of the two-point function, is symmetric for Type I AGSM fields, and this symmetry is preserved upon action by any FOS. It should however be noted that $\underline{K}$ is not required to be symmetric for a general AGSM field. ${ }^{5,13}$

While this application of the geometrical results of Secs. III and IV to optics involves $n=2$, we can easily see that $\mathrm{G}$ matrices of the form $\boldsymbol{\kappa} \underline{G}(\underline{U}, \underline{V})$ for general $n$ are what occur in the Wigner-Moyal descriptions of the thermal states of an n-dimensional isotropic oscillator, and all $S p(2 n, \mathbb{R})$ transforms of such states.

\section{CONCLUDING REMARKS}

We have presented a complete analysis of the action of symplectic transformations on Gaussian pure states in quantum mechanics. By connecting up this problem to properties of and motions on a special orbit in the Lie algebra of $\mathbf{S p}(2 n, \mathbb{R})$, it has been possible to present this action in an elegant geometrical form and also in a classical phase-space framework. It is worth remarking that the mapping $(\underline{U}, \underline{V}) \rightarrow\left(\underline{U}^{\prime}, \underline{V}^{\prime}\right)$, induced by $S \in \operatorname{Sp}(2 n, \mathbb{R})$ and given by Eq. (3.27), is, for any $S$, a canonical transformation with respect to the Poisson brackets (4.20) which, on $\widetilde{\mathcal{O}}$, have the usual canonical form (4.25). In this context we may point out the special significance of this "abcd law" which has been established here in matrix form. The original "abcd law" due to Kogelnik ${ }^{22}$ referred to the action of axially symmetric first-order optical systems, which correspond to elements of $\operatorname{SL}(2, \mathbb{R})$, on axially symmetric coherent Gaussian beams (i.e., to the $n=1, \kappa=1$ case).

For a coherent Gaussian beam of wave number $\boldsymbol{k}$ propagating along the $z$ direction, the field distribution in any transverse plane is of the form

$$
\begin{aligned}
& \psi(x, y ; q)=(2 / 7)^{1 / 2} \sigma^{-1} \exp \left[\mathrm{ik}\left(x^{2}+y^{2}\right) / 2 q\right], \\
& q^{-1}=R^{-1}+2 i / k \sigma .
\end{aligned}
$$

Clearly $\boldsymbol{R}$ is the radius of curvature (of the phase front) and $\sigma$ is the spot size (beam width) in that plane. The parameter $q$ is known as the complex radius of curvature. ${ }^{10.22}$ Kogelnik's original "abcd law" showed that under the action of a FOS

$$
\underline{S}=\left(\begin{array}{ll}
a & b \\
c & d
\end{array}\right) \in \operatorname{SL}(2, \mathbb{R}),
$$

the Gaussian nature itself is preserved and only the com- plex radius of curvature changes in the following simple way:

$$
\begin{aligned}
& S: \quad \psi(x, y ; q) \rightarrow \psi\left(x, y ; q^{\prime}\right), \\
& q^{\prime}=(a q+b) /(c q+d) .
\end{aligned}
$$

We have elsewhere generalized this to cover the action of such optical systems on partially coherent Gaussian Schell-model fields of both isotropic ${ }^{15}$ and anisotropic ${ }^{13}$ types. The results $(3.27)$ and $\left(3.27^{\circ}\right)$ can be viewed as a grand generalization of the "abcd law" (6.3) to n dimensions and in matrix form. [In fact, we have a further generalization to mixed states (for any $n$ ) in Eq. (5.8), since 6, corresponds to pure states if and only if $\kappa=1$.] A particular case of this, corresponding to solving the Schrodinger equation for GPS's with a specific Hamiltonian operator, has been presented earlier by Birula. ${ }^{2}$ For his Hamiltonian,

$$
\begin{aligned}
\underline{S}(t) & =\left[\begin{array}{ll}
\underline{\underline{a}}(t) & \underline{b}(t) \\
\vec{k}(t) & \underline{d}(t)
\end{array}\right] \\
& =\left[\begin{array}{cc}
\cos (\underline{\Omega} t) & -\underline{\Omega}^{-1} \sin (\underline{\Omega} t) \\
\underline{\Omega} \sin (\Omega t) & \cos (\underline{\Omega} t)
\end{array}\right],
\end{aligned}
$$

and by noting that $\underline{\Omega}$ is symmetric and that his $i \underline{K}$ is the negative of our $\underline{Z}=\underline{V}-\boldsymbol{i} \underline{U}$, it is readily seen that our "abcd law" (3.27)indeed gives his Eq. (2.27).

For $n=2$, the matrix "abcd law" applies to the action of a general anisotropic FOS on an AGSM field. It is quite remarkable that the "abcd law" holds for any $S \in \operatorname{Sp}(2 n, \mathbb{R})$.

From the mathematical point of view, the law (3.27) is quite significant. It is well known that the group $\mathrm{SL}(2, \mathbb{R})$ acts as a group of point transformations - the Mobius transformations - on a complex half-plane, for instance the lower half-plane. This action is closely connected with the existence of the discrete series of unitary irreducible representations of $\mathrm{SL}(2, \mathbb{R}){ }^{23}$ In contrast, the group $\operatorname{SL}(2, \mathbb{C})$ neither possesses such an action nor such unitary representations. Since $S L(2, \mathbb{R})=\operatorname{Sp}(2, \mathbb{R})$ $=\mathbf{S U}(1,1)$, it is a priori not clear in which direction a generalization might exist. Our result gives a matrix generalization of the Mobius transformations for the symplectic group $S p(2 n, \mathbb{R})$. The lower half of the complex plane for one complex variable is replaced here by the family of complex symmetric matrices $\underline{Z}=\underline{V}-i \underline{\underline{U}}$ with $\underline{U}$ being positive definite. And the easiest way to see that $\underline{\underline{Z}}^{\prime}$ obtained from $\underline{\boldsymbol{Z}}$ by Eq. (3.27) is of the same nature seems to be via our analysis of the special orbit $\mathcal{O}$ in $\operatorname{sp}(2 n, \mathbb{R})$ given in Sec. IV. Further implications of the existence of such an action will be studied elsewhere.

Finally we note that $\underline{G}^{-1}(\underline{U}, \underline{V}) \equiv \underline{G}\left(\underline{U}^{-1},-\underline{V}\right)$ is the matrix of second moments (fluctuations) in the canonical variables, as can be seen from Eq. (2.31), and from Eq. (2.28) it follows that the evolution of these fluctuations under the action of any quadratic Hamiltonian is governed by

$\mathbf{G}^{-1}(\underline{U}(t), \underline{V}(t))=\underline{S}^{T}(t) \underline{G}{ }^{-1}(\underline{U}(0), \underline{V}(0)) \underline{S}(t)$.

in fact, this evolution equation is valid for the second 
moments in any state, provided that $\underline{G}^{-1}(\underline{U}, \underline{V})$ is replaced by the matrix of second moments in that state. Since it is the dynamics of these fluctuations which is of concern in the study of squeezed states, it follows that our results are of relevance to squeezing in multimode systems. $^{24}$

As noted earlier, GPS's for $\boldsymbol{n}=1,2$ and in particular squeezed coherent states for single-mode and two-mode systems have been studied from a different point of view in Ref. 4. We further note that the squeeze operators are a subset of the elements we have studied. Since our "abcd law" in Eq. (3.27) and the equivalent formulation in Sec. IV of the quantum evolution of GPS's as classical
Hamiltonian evolution on the special orbit in the Lie algebra of $\operatorname{Sp}(2 n, \mathbb{R})$ as phase space are valid for arbitrary $n$, it will be desirable to have a detailed analysis of squeezing and the evolution of squeezed states in n-mode systems from the point of view of these formalisms; we shall return to it elsewhere.

\section{ACKNOWLEDGMENT}

This research was partially supported by the U. S. Department of Energy, under Contract No. DE-ASO576ER03992 at the University of Texas at Austin.
'Permanent address: Institute of Mathematical Sciences, Madras 600 113, Tamilnadu, India.

$\dagger$ Also at Centre for Theoretical Studies, Indian Institute of Science, Bangalore 560012, India and at Institute of Mathematical Sciences, Madras 600 113, Tamilnadu, India.

${ }^{1}$ I. Bialynicki-Birula and J. Mycielski, Ann. Phys. (N.Y.) 100, 62 (1976); Phys. Scr. 20, 539 (1979).

${ }^{2}$ I. Bialynicki-Birula, in Coherence, Cooperation and Fluctuations, edited by F. Haake, L. M. Narducci, and D. F. Walls (Cambridge University Press, London, 1986).

${ }^{3}$ D. Stoler, Phys. Rev. D 1, 3217 (1970); H. P. Yuen, Phys. Rev. A 13, 2226 (1976);J. N. Hollenhorst, Phys. Rev. D 19, 1669 (1979);D. F. Walls, Nature 306, 141 (1983).

${ }^{4}$ B. L. Schumaker, Phys. Rep. 135, 317 (1986). The term "Gaussian pure states" was used for the first time in this paper.

${ }^{5}$ R. Simon, E. C. G. Sudarshan, and N. Mukunda, Phys. Rev. A 36, 3868 (1987).

${ }^{6}$ E. P. Wigner, Phys. Rev. 40, 749 (1932); J. E. Moyal, Proc. Cambridge Philos. Soc. 45, 99 (1949); H. Weyl, The Theory of Groups and Quantum Mechanics (Dover, New York, 1931). For recent reviews on Wigner-Moyal methods see N. L. Balazs and B. K. Jennings, Phys. Rep. 104, 347 (1984); M. Hillery, R. F. O'Connell, M. O. Scully, and E. P. Wigner, ibid. 106, 121 (1984).

'Several aspects of Gaussian pure states have been studied from a different point of view in R. G. Littlejohn, Phys. Rep. 138, 193 (1986); and in B. L. Schumaker, ibid. 135, 317 (1986). Further references can be found in these papers.

${ }^{8}$ N. Mukunda, Pramana 11, 1 (1978).

${ }^{9}$ See, for example, E. C. G. Sudarshan and N. Mukunda, Classical Dynamics: A Modern Perspective (Wiley, New York, 1974).

${ }^{10}$ Coherent Gaussian laser beams are reviewed in H. Kogelnik and T. Li, Proc. IEEE 54, 1312 (1966). See also J. A. Arnand and H. Kogelnik, Appl. Opt. 8, 1687 (1969).

11J. Williamson, Am. J. Math. 58, 141 (1936);See also V. I. Arnold, Mathematical Methods of Classical Mechanics (Springer-Verlag, New York, 1978), Appendix 6.

${ }^{12}$ See, for instance, B. Y. Chu, Trans. Am. Math. Soc. 197, 45 (1974).
${ }^{13}$ R. Simon, E. C. G. Sudarshan, and N. Mukunda, Phys. Rev. A 31, 2419 (1985).

14J. T. Foley and M. S. Zubairy, Opt. Commun. 26, 297 (1978); E. Wolf and E. Collett, ibid. 25, 293 (1978);W. H. Carter and M. Bertolotti, J. Opt. Soc. Am. 68, 329 (1978); B. E. A. Saleh, ibid. 30, 135 (1979);F. Gori, ibid. 41, 383 (1982); A. T. Friberg and R. J. Sudol, ibid. 41, 383 (1982); A. Starikov and E. Wolf, J. Opt. Soc. Am. 72, 923 (1982); F. Gori, Opt. Commun. 46, 149 (1983); F. Gori and R. Grella, ibid. 49, 173 (1984).

${ }^{15} \mathrm{GSM}$ beams have been studied from the present point of view in R. Simon, E. C. G. Sudarshan, and N. Mukunda, Phys. Rev. A 29, 3273 (1984).

16Whereas the full set of AGSM fields studied in Ref. 13 constitutes a ten-parameter family, a restricted class of such fields has been analyzed in Y. Li and E. Wolf, Opt. Lett. 7, 256 (1982); F. Gori and G. Gauttari, Opt. Commun. 48, 7 (1983); R. Simon, J. Opt. 14, 92 (1985); P. De Santis, F. Gori, G. Gauttari, and C. Palma, Opt. Acta 33, 315 (1986).

${ }^{17}$ L. Mandel and E. Wolf, Rev. Mod. Phys. 37, 231 (1965).

${ }^{18}$ H. Bacry and M. Cadilhac, Phys. Rev. A 23, 2533 (1981); M. Nazarathy and J. Shamir, J. Opt. Soc. Am. 72, 356 (1982); R. Simon, E. C. G. Sudarshan, and N. Mukunda, Phys. Rev. A 31,2419 (1985).

${ }^{19}$ E. C. G. Sudarshan, N. Mukunda, and R. Simon, Opt. Acta 32, 855 (1985).

${ }^{20}$ Whereas the Wigner-Moyal transform of the density matrix in quantum mechanics is called the Wigner distribution, that of the cross-spectral density in optics is called the Wolf function; E. C. G. Sudarshan, Phys. Lett. 73A, 269 (1979); Physica 96A, 315 (1979); Phys. Rev. A 23, 2802 (1981);R. Simon, Pra. mana 20, 105 (1982).

${ }^{21}$ R. Simon, Opt. Commun. 42, 293 (1983);55, 381 (1985).

${ }^{22}$ H. Kogelnik, Bell Sys. Tech. J. 44, 455 (1965), See also, D. Marcuse, Light Transmission Optics (Van Nostrand Reinhold, New York, 1972).

${ }^{23}$ See, for instance, I. M. Gel'fand, M. I. Graev, and N. Ya. Vilenkin, Generalized Functions (Academic, New York, 1966), Vol. 5.

${ }^{24}$ M. J. Milburn, J. Phys. A 17, 737 (1984);B. L. Schumaker, Phys. Rep. 135, 317 (1986) 